\title{
Bioassay development for ultrasensitive detection of influenza $A$ nucleoprotein using digital ELISA
}

\author{
Karen Leirs ${ }^{\mathrm{a}}$, Phalguni Tewari Kumar ${ }^{\mathrm{a}}$, Deborah Decrop ${ }^{\mathrm{a}}$, Elena Pérez-Ruiz ${ }^{\mathrm{a}}$, Pelin Leblebici ${ }^{\mathrm{a}}$, Bram Van $^{2}$ \\ Kelst $^{a}$, Griet Compernolle ${ }^{b}$, Hanne Meeuws ${ }^{c}$, Liesbeth Van Wesenbeeck ${ }^{c}$, Ole Lagatie ${ }^{c}$, Lieven \\ Stuyver ${ }^{c}$, Ann Gils ${ }^{b}$, Jeroen Lammertyn ${ }^{a *}$ and Dragana Spasic ${ }^{a}$ \\ ${ }^{a}$ BIOSYST-MeBioS, KU Leuven, Belgium \\ baboratory for Therapeutic and Diagnostic Antibodies, KU Leuven, Belgium \\ cJanssen Diagnostics, Beerse, Belgium \\ *corresponding author: jeroen.lammertyn@kuleuven.be
}

\section{Screening of the binding of antibodies with immobilized nucleoprotein using ELISA}

To decide upon which antibodies to use for the development of a sensitive bioassay for influenza $A$ nucleoprotein detection using digital ELISA, the binding performance of seven selected commercial antibodies (monoclonal: SinoM, Ab11, Ab12, Ab66 and Ab67 and polyclonal: SinoP and ImP) was first investigated using standard ELISA with recombinant nucleoprotein immobilized directly on the microtiter plate surface. A volume of $200 \mu \mathrm{L}$ of $4 \mu \mathrm{g} / \mathrm{mL}$ nucleoprotein diluted in PBS buffer, was added to each well and incubated for $72 \mathrm{~h}$ at $4{ }^{\circ} \mathrm{C}$. Following incubation, the plate was blocked with $200 \mu \mathrm{L}$ of $1 \% \mathrm{BSA}$ solution in PBS for $2 \mathrm{~h}$ at room temperature (RT). Next, the plate was washed six times with PBS containing $0.002 \% \mathrm{v} / \mathrm{v}$ Tween 80 , followed by applying $180 \mu \mathrm{L}$ of antibody solution diluted in PTA buffer (PBS, 0.1\% BSA, 0.002\% Tween 80). Two antibody concentrations ( 80 and $3.2 \mathrm{ng} / \mathrm{mL}$ ) were tested. Incubation with antibody solutions was performed for $1 \mathrm{~h}$ at RT and overnight at $4{ }^{\circ} \mathrm{C}$, to evaluate the effect of incubation time. After incubation, the plate was washed as mentioned before and incubated with $170 \mu \mathrm{L}$ of detection antibody for $2 \mathrm{~h}$ at RT. HRP-labeled rabbit anti-mouse (RAM) or goat anti-rabbit (GAR1) were used as detection antibodies, for detecting monoclonal and polyclonal antibodies, respectively. According to manufacturer guidelines, 1/10000 dilution for RAM and 1/2000 dilution for GAR1 were prepared from the stock solution in PTA buffer. After incubation, the plate was washed and color was generated using $160 \mu \mathrm{L}$ of $0.4 \mathrm{mg} / \mathrm{mL} \mathrm{o-}$ phenylenediamine with $\mathrm{H}_{2} \mathrm{O}_{2}$ in $0.1 \mathrm{M}$ sodium citrate and $0.2 \mathrm{M}$ di-sodium phosphate solution, $\mathrm{pH} 5$. The reaction was stopped after 10 min by adding $50 \mu \mathrm{L}$ of $4 \mathrm{M} \mathrm{H}_{2} \mathrm{SO}_{4}$ and the absorbance was measured at $492 \mathrm{~nm}$. The measured absorbance was corrected for the background signal generated due to aspecific interactions of the HRP-labeled antibodies with the nucleoprotein coated on the microtiter plate surface.

The assay layout and the results of the ELISA screening are shown in Figure S1. All tested antibodies were able to bind to immobilized nucleoprotein when applying an antibody concentration of $80 \mathrm{ng} / \mathrm{mL}$ and incubating overnight. Although most of the antibodies persisted well shortening the incubation time from overnight to $1 \mathrm{~h}$ (with a drop in signal varying between $4 \%$ and $26 \%$ for SinoM, Ab11, Ab12, Ab66 and SinoP), two antibodies, namely Ab67 and ImP, showed a substantially higher reduction in signal of $56 \%$ and $48 \%$, respectively. Applying a lower antibody concentration $(3.2 \mathrm{ng} / \mathrm{mL})$, caused a significant drop in signal for most antibodies (more than $71 \%$ ) compared to the 
higher concentration tested, irrespective of the assay time. The only antibody that endured this assay condition was Ab11 with a drop of only $45 \%$ for overnight incubation. SinoM, Ab67 and ImP showed almost no reactivity after $1 \mathrm{~h}$ incubation time at low concentration and were therefore excluded from sandwich ELISA tests. Moreover, since the aim was to explore the possibilities of sensitive detection of influenza A nucleoprotein and SinoP was percentage-wise more severely affected by lowering the antibody concentration compared to monoclonal antibodies, it was also not explored further as capture antibody in the ELISA experiments. The variation in the reactivity of the antibodies can be explained by the different types of interactions that occur between the antibody and the target, such as Van der Waals and hydrogen bonds. Depending on the type of the interaction, the binding is differently influenced when changing the incubation conditions ${ }^{1}$. In conclusion, Ab11, Ab12 and $A b 66$ were taken further for testing their binding performance towards free nucleoprotein in a sandwich ELISA.

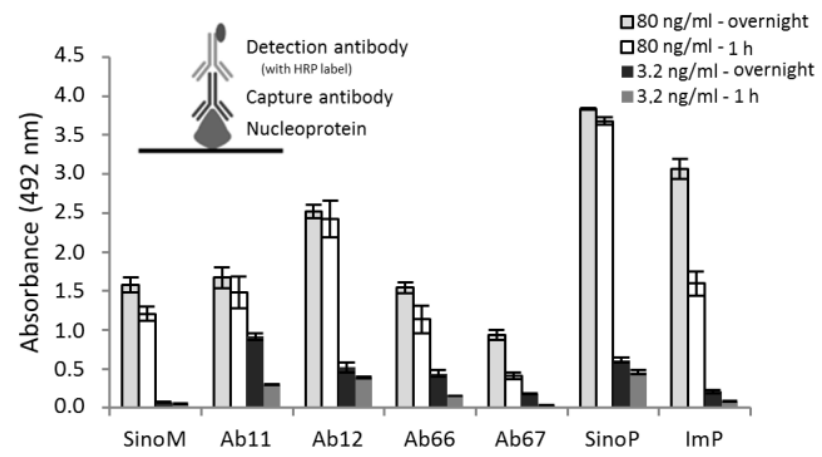

Figure S1: Results of the binding capacity of the different antibodies when recombinant influenza A nucleoprotein was immobilized on the surface and binding was determined using both an overnight as well as a $1 \mathrm{~h}$ incubation time. Error bars are standard deviations based on three repetitions.

\section{Selection of secondary and detection antibody pair for sandwich ELISA}

To detect nucleoprotein in solution, the capture antibody is immobilized on the microtiter plate surface and a secondary and detection antibody are used to generate the signal. In order to obtain the most optimal pair of secondary and detection antibody, which gives high signal-to-noise ratio, several control experiments were performed. First, the effect of the detection antibody was analyzed by testing GAR1 and GAR2 in an assay where both nucleoprotein and secondary antibody were omitted. The results are shown in Table S1. GAR2 gave lower background signal than GAR1 when used in combination with Ab11, Ab12 and Ab66. Therefore, GAR2 antibody was used in further experiments.

Table S1: Influence of the detection antibody on the background signal. Absorbance values measured at $492 \mathrm{~nm}$ are given. The errors shown in brackets are standard deviations $(n=3)$.

\begin{tabular}{ccc} 
Capture antibody & Detection antibody & Absorbance \\
\hline Ab11 & GAR 1 & $\mathbf{0 . 1 3 9 ( \pm 0 . 0 0 7 )}$ \\
& GAR 2 & $\mathbf{0 . 0 1 8}( \pm 0.006)$ \\
Ab12 & GAR 1 & $\mathbf{0 . 1 0 8}( \pm 0.002)$ \\
& GAR 2 & $\mathbf{0 . 0 3 8}( \pm 0.002)$ \\
Ab66 & GAR 1 & $\mathbf{0 . 0 3 1}( \pm 0.019)$ \\
& GAR 2 & $\mathbf{0 . 0 1 4}( \pm 0.003)$
\end{tabular}

\footnotetext{
${ }^{1}$ Reverberi, R.; Reverberi, L. 2007. Blood Transfus. 5, 227-240.
} 
Next, the influence of the secondary antibody on the reactivity of the capture antibody (Ab11, Ab12 or Ab66) was evaluated. Polyclonal antibodies are chosen as secondary antibodies for practical reason - since a detection antibody will be used, the secondary antibody should be generated in a different host species compared to the capture antibody in order to avoid unspecific signal generation. Two polyclonal antibodies (SinoP and ImP) were tested as secondary antibodies in the absence of nucleoprotein. The results of this test are shown in Table S2. Because of the lower background signal and smaller standard deviation, SinoP was chosen as secondary antibody.

Table S2: Influence of the secondary antibody on the background signal. Absorbance values measured at $492 \mathrm{~nm}$ are given. The errors shown in brackets are standard deviations $(n=3)$.

\begin{tabular}{ccc} 
Capture antibody & Secondary antibody & Absorbance \\
\hline Ab11 & ImP & $\mathbf{0 . 2 2 3}( \pm 0.012)$ \\
& SinoP & $\mathbf{0 . 0 6 3}( \pm 0.003)$ \\
Ab12 & $\operatorname{ImP}$ & $\mathbf{0 . 1 1 8}( \pm 0.036)$ \\
& SinoP & $\mathbf{0 . 1 0 5}( \pm 0.004)$ \\
Ab66 & ImP & $\mathbf{0 . 0 8 9}( \pm 0.002)$ \\
& SinoP & $\mathbf{0 . 0 2 8}( \pm 0.001)$
\end{tabular}

\section{Detection of free nucleoprotein using sandwich ELISA}

After establishing the most optimal pair of secondary and detection antibody, different concentrations of recombinant nucleoprotein $(0.128-80 \mathrm{ng} / \mathrm{mL}$, five-fold dilutions) were detected using ELISA. The target was incubated with the capture antibody overnight or for $1 \mathrm{~h}$. The assay layout and the obtained calibration curves are shown in Figure S2. The linear parts of the calibration curves and explanation of the results are given in the article.
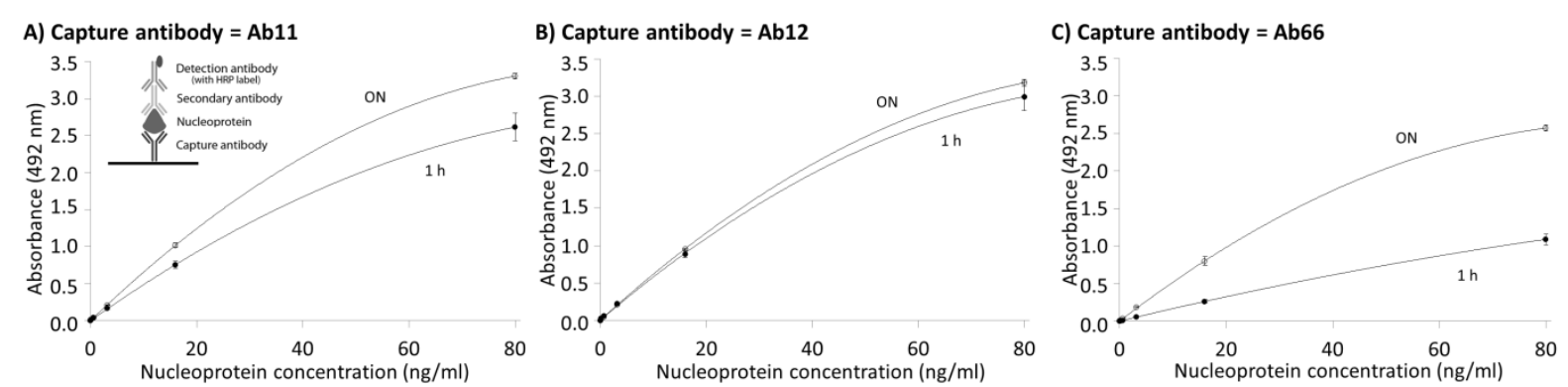

Figure S2: Reactivity of the capture antibodies Ab11 (A), Ab12 (B) and Ab66 (C) towards recombinant influenza $A$ nucleoprotein during overnight (ON) and $1 \mathrm{~h}$ incubation. Absorbance $(492 \mathrm{~nm})$ was corrected for background signal. For each antibody, several concentrations of nucleoprotein were tested $(0.128-80 \mathrm{ng} / \mathrm{mL})$ and the graphs show the complete calibration curve with a trend line indicating a saturation behavior. Error bars are standard deviations based on three repetitions.

\section{Antibody screening using SPR}

All antibodies were also challenged using an SPR system, i.e. Biacore 3000 and screening was performed following two approaches. First, recombinant nucleoprotein was coupled to the CM5 chip surface and $500 \mathrm{nM}$ of each antibody was used as analyte. Second, each antibody was coupled to the surface and $500 \mathrm{nM}$ recombinant nucleoprotein was used as analyte. The interaction time between 
the nucleoprotein and the antibody was 6 min for both configurations and the results of these interactions are shown in Table S3.

Table S3: Screening of the interaction between the antibodies and recombinant influenza A nucleoprotein using SPR. Either nucleoprotein or antibody was immobilized on the sensor surface and subsequently $500 \mathrm{nM}$ of antibody or nucleoprotein (diluted in buffer) was injected, respectively. The measured interaction between the antibodies and nucleoprotein is given as response units (RU). Antibodies used further for determining affinity constants are indicated in bold.

\begin{tabular}{ccc}
$\begin{array}{c}\text { Antibody } \\
\text { name }\end{array}$ & $\begin{array}{c}\text { Antibody in } \\
\text { solution (RU) }\end{array}$ & $\begin{array}{c}\text { Antibody } \\
\text { coupled (RU) }\end{array}$ \\
\hline ImP & 10.3 & 11.5 \\
SinoP & 3.3 & $\mathbf{2 6 . 7}$ \\
SinoM & 0.2 & 4.6 \\
Ab66 & 0 & 332.1 \\
Ab11 & 0 & 46.5 \\
Ab67 & 0 & 4.7 \\
Ab12 & 0 & $\mathbf{2 2 . 9}$
\end{tabular}

Substantial differences were observed among different antibodies when nucleoprotein was immobilized on the surface (Table S3) and the differences were more pronounced than those seen in the similar assay configuration when performing ELISA experiment (Figure S1). Only two polyclonal antibodies (ImP and SinoP) showed some interaction with the immobilized nucleoprotein, whereas the signal for all monoclonal antibodies was below the limit of detection. We suspect this can be explained by the fact that polyclonal antibodies recognize multiple epitopes, thus better tolerating if one of the epitopes of the target is blocked by its orientation on the chip surface ${ }^{2}$, which might become a crucial advantage when the time for interaction with the target is limited, like in the case of flow-based SPR systems. Based on rather limited interaction with the free recombinant nucleoprotein on the SPR platform and the obtained results from the ELISA experiments, three antibodies (SinoM, Ab67 and ImP) were excluded from further testing.

\section{Selection of fitting models for determining antibody affinity constants using SPR}

To determine the affinity constants of the tested antibodies, the obtained SPR curves were fitted with a 1:1 Langmuir model using Biacore Evaluation Software version 4.1. For Ab66, SinoP and Ab12, the fitted curves corresponded well with the experimental curves (Figure S3 A, B and C). However, the obtained experimental curves for Ab11 could not be fitted using the Langmuir model. Due to the possibility of nucleoprotein to form multimers, the multimer can orient in such a way that it is able to bind to two antibodies. This is more pronounced for antibodies with a higher dissociation rate or if the epitopes are located further away from the binding sites of two nucleoprotein monomers. Therefore, the bivalent analyte model was used for fitting the experimental curves obtained for Ab11 (Figure S3 D), since this model describes a necessary initial interaction of the multimeric analyte with a receptor (confer Langmuir model) and an optional subsequent interaction of the same analyte molecule with another receptor (Biacore assay handbook).

\footnotetext{
${ }^{2}$ Lipman, N. S.; Jackson, L. R.; Trudel, L. J.; Weis-Garcia, F. 2005. ILAR J. 46, 258-268.
} 

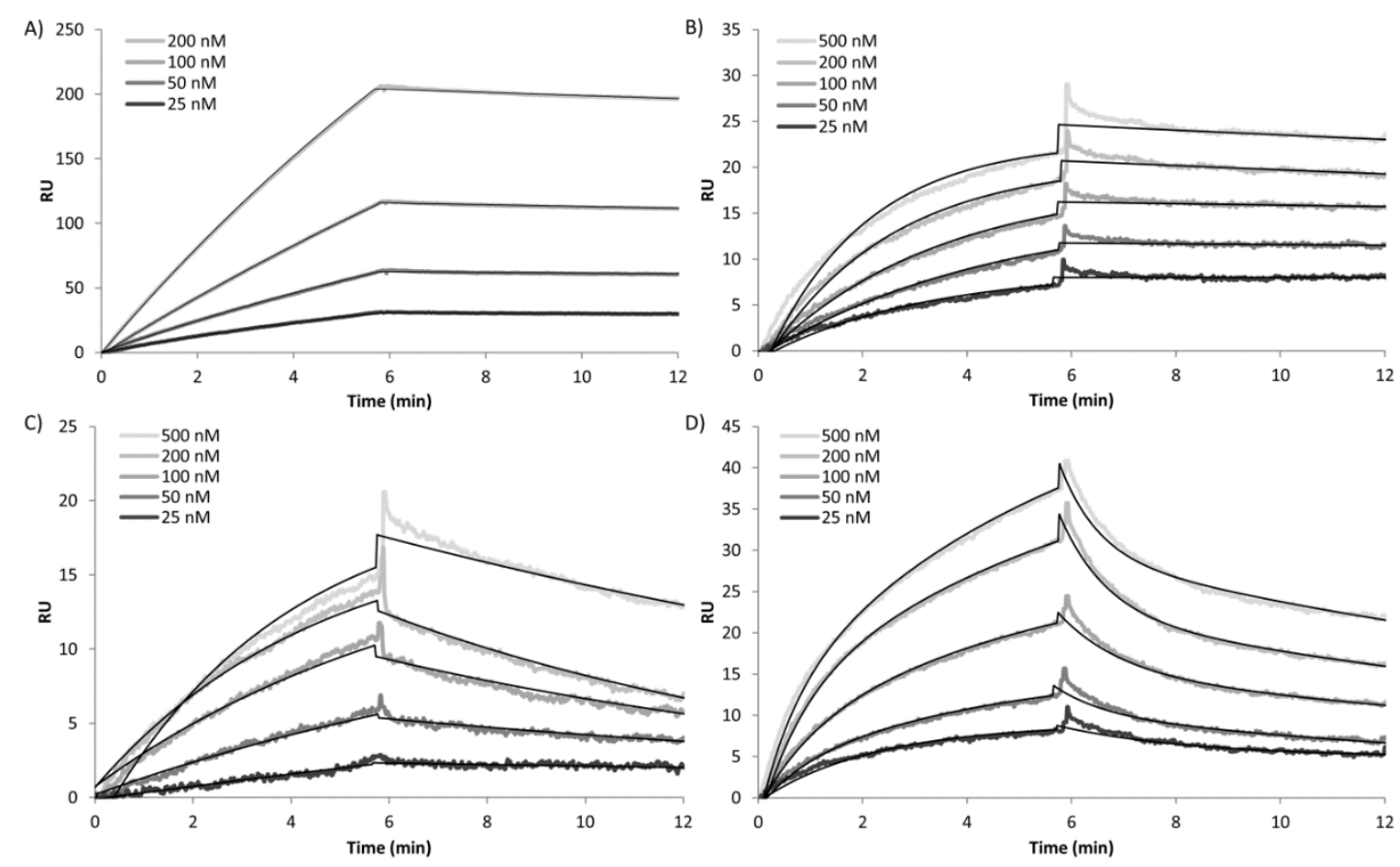

Figure S3: Association and dissociation curves and their corresponding fitting for Ab66 (A), SinoP (B), Ab12 (C) and Ab11 (D). For Ab66, SinoP and Ab12, a 1:1 Langmuir model was used and for Ab11 the fitting is based on a bivalent analyte model.

\section{Determination of lysis buffer concentration}

The lysis capability of the chosen detergent (NLS) was tested for two concentrations ( $0.5 \%$ and $0.1 \%)$. The amount of released nucleoprotein was determined using a sandwich ELISA with Ab11 as capture antibody, SinoP as secondary, and GAR2 as detection antibody. Multiple dilutions of purified virus were incubated for $1 \mathrm{~h}$ on the ELISA plate in the presence of NLS, to allow simultaneous release and capture of the nucleoprotein. The results are shown in Figure S4, indicating no significant difference between $0.5 \%$ and $0.1 \%$ NLS. Therefore, $0.1 \%$ was used in further experiments.

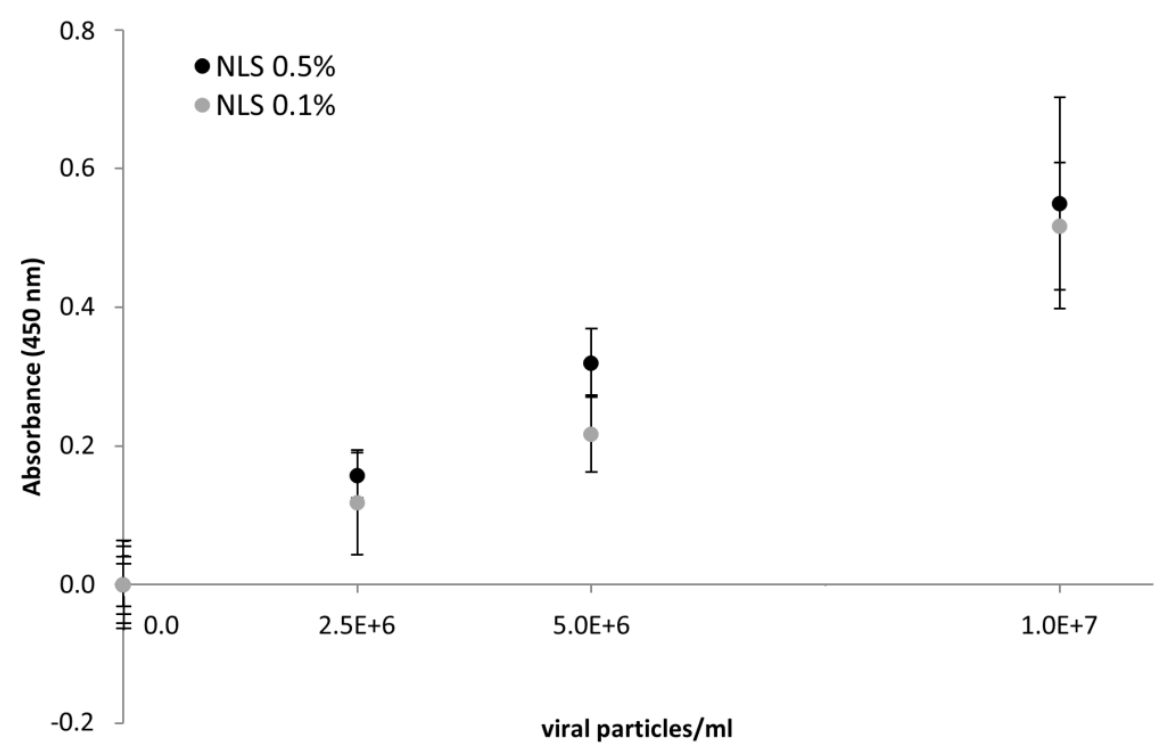

Figure S4: Lysis capability of NLS tested for two concentrations ( $0.1 \%$ and $0.5 \%)$, during $1 \mathrm{~h}$ incubation time with sandwich ELISA (capture antibody $=A b 11$, secondary antibody $=$ SinoP, detection antibody $=$ GAR2). Absorbance at $450 \mathrm{~nm}$ was measured and corrected for background signal. Error bars are standard deviations $(n=3)$. NLS clearly induced the release of nucleoprotein from the viral particles without showing significant difference between the two tested concentrations. 


\section{Detection of recombinant nucleoprotein using digital ELISA}

For the sensitive detection of recombinant nucleoprotein, an in-house developed digital ELISA system was used. A schematic representation of the assay flow is shown in Figure S5.

A

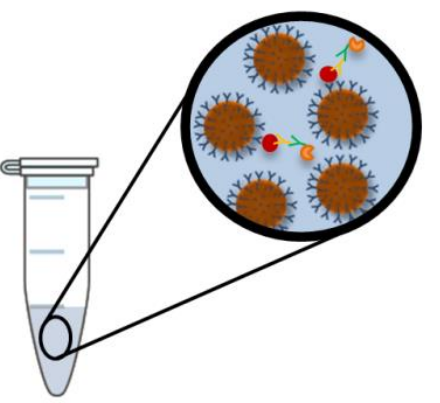

Capture of target protein on antibodyfunctionalized magnetic particles and labeling with enzyme-conjugated antibodies

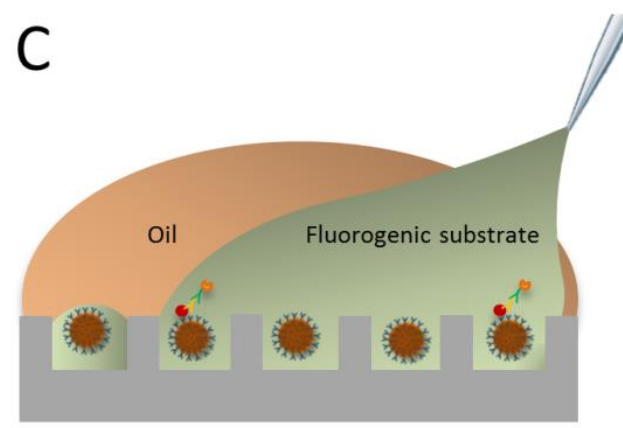

Sealing femtoliter droplets of fluorogenic substrate with oil
B

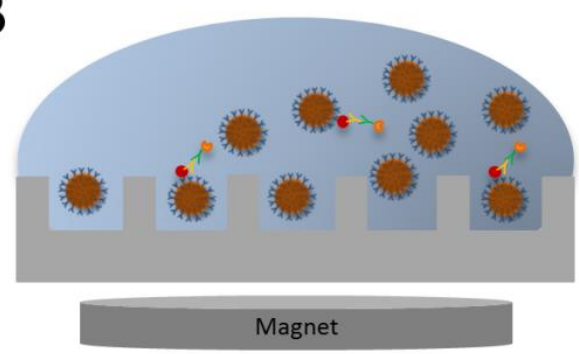

Magnet-assisted seeding of individual magnetic particles in the microwell array

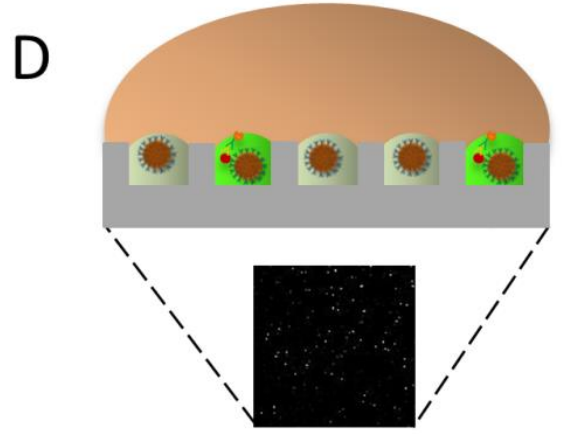

Generation of fluorescent signal and digital counting of single protein molecules

Figure S5: Assay flow of the digital ELISA for sensitive detection of recombinant influenza A nucleoprotein. A) First, nucleoprotein (represented by the red dot) is captured on superparamagnetic beads functionalized with specific antibodies (blue antibody), followed by the consecutive binding of detection (yellow antibody) and labeling antibody (green antibody with conjugated enzyme represented in orange) to form the immunocomplexes. B) Next, the beads are seeded individually in hydrophilic-in-hydrophobic microwells by transporting the droplet over the microwell array that is positioned above a magnet. C) Fluorogenic substrate is added and the microwells are sealed by applying an oil droplet, creating femtolitersized reaction wells. D) After an incubation time, the fluorescent product generated by the enzyme-conjugated labeling antibody is visualized using a microscope. 